\title{
A Route to Integrating Dynamic 4D X-ray Computed Tomography and Machine Learning to Model Material Performance
}

\author{
Nikolaus L. Cordes ${ }^{1}$, Kevin Henderson ${ }^{1}$ and Brian M. Patterson ${ }^{1}$ \\ 1. Engineered Materials, Materials Science \& Technology Division, Los Alamos National Laboratory, \\ Los Alamos, NM, USA
}

Machine learning has recently been implemented in materials science where it has opened new pathways to modeling and predicting material performance [1]. A material property that is difficult to model and predict (as well as difficult to tailor during material fabrication) is the compressive performance of polymeric foams. These materials are often used in applications where high strength, light weight, low density and/or low cost components are desired and their compressive properties are often essential to the intended application. However, a population of polymer foams samples with analogous polymeric material properties may exhibit extremely different compressive stress-strain curves which can be attributed to the various micrometer-scale void morphologies present in the population. By capturing these void microstructures at various stress-strain states via dynamic 4D (i.e., 3D + time) X-ray computed tomography (X-ray CT), quantitative void microstructure descriptors can used as inputs into machine learning algorithms for the purposes of developing a polymeric foam compressive performance model. This will allow a deeper understanding of exactly how the void microstructure affects a polymer foam's compressive response in a mathematical framework as well as establish a methodology for the study of other systems undergoing a physical dynamic external stimulus. The work presented here is a proof-of-concept study combining 4D X-ray CT data with a traditional multivariate regression technique, partial least squares regression (PLS), and a machine learning technique, Artificial Neural Network (ANN), for the purposes of modeling the stress-strain response using void microstructural information. Results of these analyses will be shown as well as a path forward for providing a robust study of this material property.

4D X-ray CT image data of polymer foams undergoing dynamic uniaxial compression was acquired at Argonne National Laboratory's Advanced Photon Source (Fig 1) with a strain rate of $\sim 10^{-2} \mathrm{~s}^{-1} .900$ radiographs were captured as the sample rotated $180^{\circ}$ over the course of $1 \mathrm{~s}$ resulting in 20 collected tomograms (i.e., 3D image data sets) over the course of $100 \mathrm{~s}$ [2]. Void microstructural information of two polymer foams, a hydrogen-blown polysiloxane foam (Fig. 1) and a urea-prilled polysiloxane foam, was captured at increasing stress-strain states, from $0 \%$ up to $60 \%$ compressive strain. This 4D data was then processed and analyzed using image analysis software then used as inputs (training and testing sets) into PLS and ANN using PLSToolbox operating in Matlab.

Stress-strain results obtained by PLS regression and an ANN, as well as the experimental stress-strain curve of the polymer foam (presented in Fig. 1), are presented in Figure 2. PLS regression, which assumes a proportional relationship between the input variables, does not adequately model the stress and strain values due to the nonlinear nature of the stress strain curve. ANN provides more adequate stress-strain values; however, this method represents a "black box" in the machine learning spectrum and subsequently does not provide any relationship information between the input variables and the output values. These results highlight the need for statistical learning methods for producing a mathematical model that can adequately describe the stress-strain curves of polymer foams using void microstructural information as inputs. 


\section{References:}

[1] T. Mueller, A. G. Kusne, R. Ramprasad in "Reviews in Computational Chemistry: Vol. 29”, ed. A. L. Parrill, K. B. Lipkowitz, (Wiley) p. 480

[2] Patterson et al., Journal of Materials Science 51(1) (2016), p. 171.
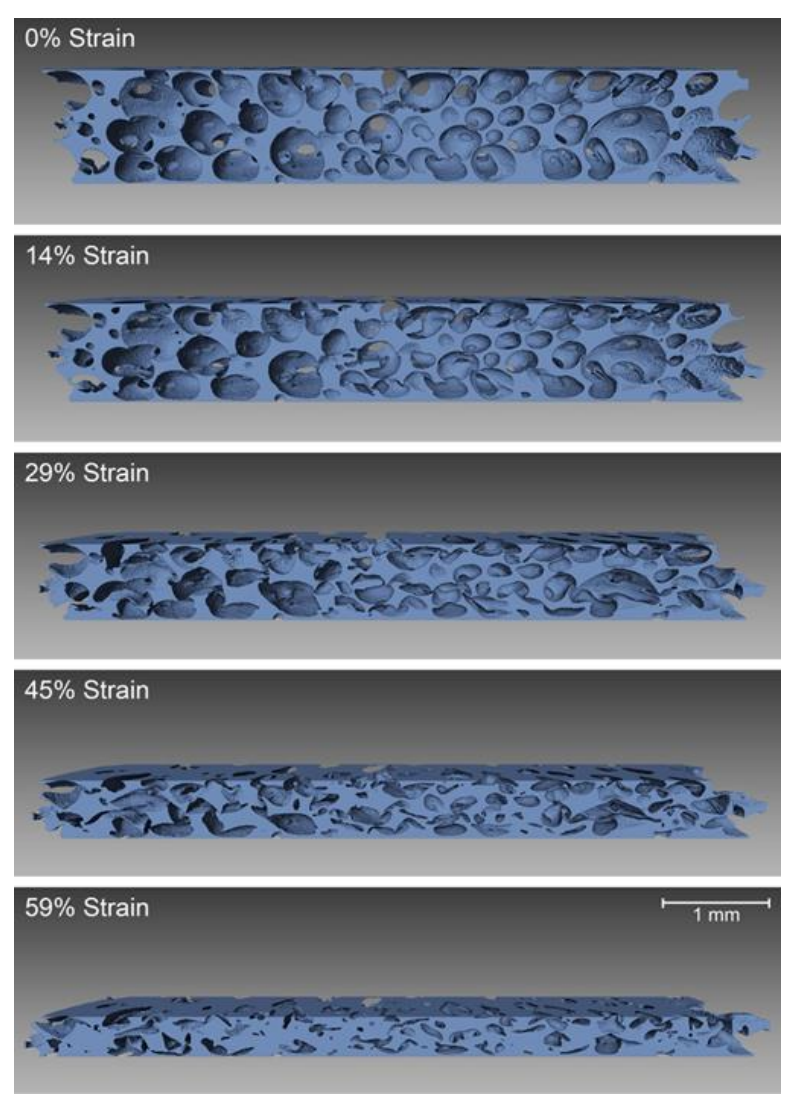

Figure 1. Volume renderings, obtained via 4D X-ray CT, of a hydrogen-blown polysiloxane foam at increasing stress-strain states.
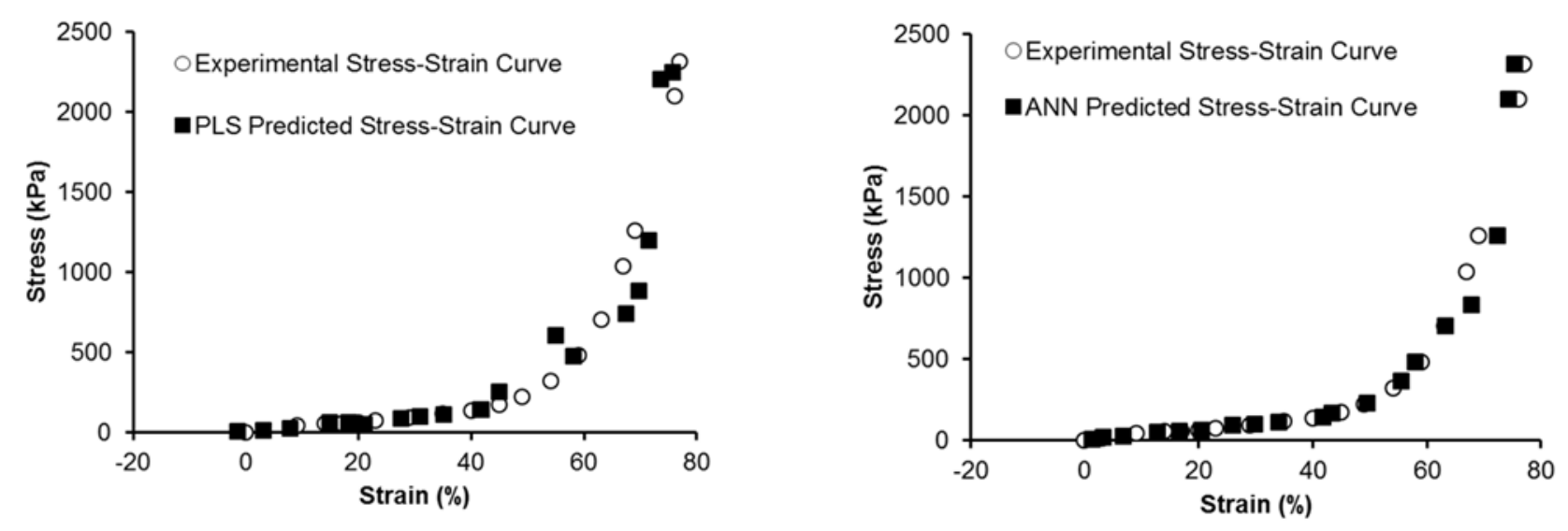

Figure 2. Predicted stress-strain curves of the polymer foam (see Fig. 1) obtained by PLS regression (left) and an ANN (right). 